Article

\title{
Further Improved Weighted Arithmetic-Geometric Operator Mean Inequalities
}

\author{
Jianming Xue ${ }^{1, *}$ and Xingkai $\mathrm{Hu}^{2,3}$ \\ 1 Oxbridge College, Kunming University of Science and Technology, Kunming 650106, China \\ 2 Faculty of Civil Engineering and Mechanics, Kunming University of Science and Technology, \\ Kunming 650500, China \\ 3 Faculty of Science, Kunming University of Science and Technology, Kunming 650500, China \\ * Correspondence: xkhu@kmust.edu.cn or xuejianming104@163.com
}

Received: 9 July 2019; Accepted: 2 September 2019; Published: 5 September 2019

\begin{abstract}
The main purpose of this paper is to present some weighted arithmetic-geometric operator mean inequalities. These inequalities are refinements and generalizations of the corresponding results. An example is provided to confirm the effectiveness of the results.
\end{abstract}

Keywords: operator inequality; positive linear map; weighted arithmetic-geometric operator mean

\section{Introduction}

It is well known that $A \geq B$ implies $A^{2} \geq B^{2}$ for some positive operators $A, B$. It is interesting to ask for what kind of operator inequalities, when they are squared, the inequality relation can be preserved. In 2013, Lin ([1], Theorem 2.8) proved that the operator Kantorovich inequality can be squared. Similarly, Lin ([2], Theorem 2.1) found that the reverse arithmetic-geometric operator mean inequalities for the Kantorovich constant can be squared:

$$
\Phi^{2}\left(\frac{A+B}{2}\right) \leq K^{2}(h) \Phi^{2}\left(A \sharp_{\frac{1}{2}} B\right)
$$

and

$$
\Phi^{2}\left(\frac{A+B}{2}\right) \leq K^{2}(h)\left[\Phi(A) \sharp_{\frac{1}{2}} \Phi(B)\right]^{2},
$$

where $0<m \leq A, B \leq M, h=\frac{M}{m}$.

Here, $K(h)=\frac{(h+1)^{2}}{4 h}$ is called the Kantorovich constant and satisfies the following properties:

(i) $K(1)=1$;

(ii) $K(h)=K\left(\frac{1}{h}\right)$ for $h>0$;

(iii) $K(h)$ is monotone increasing on $[1,+\infty)$ and monotone decreasing on $(0,1]$.

It is to be understood throughout the paper that $m, m^{\prime}, M, M^{\prime}$ present scalars. I denotes the identity operator. Let $\mathcal{B}(\mathcal{H})$ stand for the $C^{*}$-algebra of all bounded linear operators on a Hilbert space $(\mathcal{H},\langle\cdot, \cdot\rangle)$. We write $A \geq 0(A>0)$ to mean that $A$ is a positive (strictly positive) operator. A linear map $\Phi: \mathcal{B}(\mathcal{H}) \rightarrow \mathcal{B}(\mathcal{K})$ is positive if $\Phi(A) \geq 0$ whenever $A \geq 0$. It is said to be unital if $\Phi(I)=I$. The operator norm is denoted by $\|\cdot\|$. For convenience, we use the following notations to define the $\alpha$-weighted arithmetic mean and $\alpha$-weighted geometric mean of $A$ and $B$ :

$$
A \nabla_{\alpha} B=(1-\alpha) A+\alpha B, A \sharp_{\alpha} B=A^{\frac{1}{2}}\left(A^{-\frac{1}{2}} B A^{-\frac{1}{2}}\right)^{\alpha} A^{\frac{1}{2}},
$$

where $A, B>0$ and $\alpha \in[0,1]$. 
Zhang ([3], Theorem 2.6) generalized (1) and (2) to the power of $p(p \geq 2)$ as follows:

$$
\Phi^{2 p}\left(\frac{A+B}{2}\right) \leq \frac{\left[K(h)\left(M^{2}+m^{2}\right)\right]^{2 p}}{16 M^{2 p} m^{2 p}} \Phi^{2 p}\left(A_{\sharp_{1}} B\right)
$$

and

$$
\Phi^{2 p}\left(\frac{A+B}{2}\right) \leq \frac{\left[K(h)\left(M^{2}+m^{2}\right)\right]^{2 p}}{16 M^{2 p} m^{2 p}}\left[\Phi(A) \sharp_{\frac{1}{2}} \Phi(B)\right]^{2 p} .
$$

Moreover, Xue ([4], Theorem 2) derived refinements and generalizations for inequalities (1)-(2):

$$
\Phi^{2}\left(A \nabla_{\alpha} B\right) \leq\left[\frac{K(h)}{S\left(h^{\prime r}\right)}\right]^{2} \Phi^{2}\left(A \sharp_{\alpha} B\right)
$$

and

$$
\Phi^{2}\left(A \nabla_{\alpha} B\right) \leq\left[\frac{K(h)}{S\left(h^{\prime r}\right)}\right]^{2}\left[\Phi(A) \sharp_{\alpha} \Phi(B)\right]^{2},
$$

where $\alpha \in[0,1], S\left(h^{\prime}\right)=\frac{h^{\prime} \frac{1}{h^{\prime}-1}}{e \log h^{\prime} \frac{1}{h^{\prime}-1}}, h=\frac{M}{m}, h^{\prime}=\frac{M^{\prime}}{m^{\prime}}$ and $r=\min \{\alpha, 1-\alpha\}$.

For further reading related to operator inequalities, the reader is referred to recent papers [5-9], and the references therein.

Following an idea from Lin [2], we shall present some new weighted arithmetic-geometric operator mean inequalities, which can be seen as complementary to inequalities (3)-(6). Moreover, an example shows that our results are sharper than inequalities (5)-(6).

\section{Main Results}

We start this section with some basic lemmas which are important in terms of proving the main results.

Lemma 1. [10] If $A, B>0$, then

$$
\|A B\| \leq \frac{1}{4}\|A+B\|^{2} .
$$

Lemma 2. [11] If $A>0$, then for every positive unital linear map $\Phi$,

$$
\Phi\left(A^{-1}\right) \geq \Phi^{-1}(A) .
$$

Lemma 3. [12] If $A, B>0$, then

$$
\left\|A^{r}+B^{r}\right\| \leq\left\|(A+B)^{r}\right\|,
$$

where $1 \leq r<\infty$.

Lemma 4. [13] Assume that $0<m \leq A \leq m^{\prime}<M^{\prime} \leq B \leq M$ or $0<m \leq B \leq m^{\prime}<M^{\prime} \leq A \leq M$. Then, for any $\alpha \in[0,1]$,

$$
A \nabla_{\alpha} B \geq S\left(h^{\prime r}\right) A \sharp_{\alpha} B,
$$

where $S\left(h^{\prime}\right)=\frac{h^{\prime} \frac{1}{h^{\prime}-1}}{e \log h^{\prime} h^{\prime}-1}, h^{\prime}=\frac{M^{\prime}}{m^{\prime}}$ and $r=\min \{\alpha, 1-\alpha\}$.

It is easy to see that

$$
A^{-1} \nabla_{\alpha} B^{-1} \geq S\left(h^{\prime r}\right) A^{-1} \sharp_{\alpha} B^{-1} .
$$


Since $0<m \leq A \leq m^{\prime}<M^{\prime} \leq B \leq M$ or $0<m \leq B \leq m^{\prime}<M^{\prime} \leq A \leq M$, it follows that

$$
\frac{1}{M}<B^{-1}<\frac{1}{M^{\prime}}<\frac{1}{m^{\prime}}<A^{-1}<\frac{1}{m}
$$

or

$$
\frac{1}{M}<A^{-1}<\frac{1}{M^{\prime}}<\frac{1}{m^{\prime}}<B^{-1}<\frac{1}{m} .
$$

By Lemma 4, we have inequality (10).

Theorem 1. Assume $A, B>0$ and let $\Phi$ be a positive unital linear map. If $0<m \leq A \leq m^{\prime}<M^{\prime} \leq B \leq M$ or $0<m \leq B \leq m^{\prime}<M^{\prime} \leq A \leq M$, then for any $\alpha \in[0,1]$,

$$
\begin{aligned}
& {\left[\Phi\left(A \nabla_{\alpha} B\right)+M m\left(\Phi\left(A^{-1} \nabla_{\alpha} B^{-1}\right)-S\left(h^{\prime r}\right) \Phi\left(A^{-1} \sharp_{\alpha} B^{-1}\right)\right)\right]^{2}} \\
& \leq\left[\frac{K(h)}{S\left(h^{\prime r}\right)}\right]^{2} \Phi^{2}\left(A \sharp_{\alpha} B\right)
\end{aligned}
$$

and

$$
\begin{aligned}
& {\left[\Phi\left(A \nabla_{\alpha} B\right)+M m\left(\Phi\left(A^{-1} \nabla_{\alpha} B^{-1}\right)-S\left(h^{\prime r}\right) \Phi\left(A^{-1}\right) \sharp_{\alpha} \Phi\left(B^{-1}\right)\right)\right]^{2}} \\
& \leq\left[\frac{K(h)}{S\left(h^{\prime r}\right)}\right]^{2}\left[\Phi(A) \sharp_{\alpha} \Phi(B)\right]^{2},
\end{aligned}
$$

where $S\left(h^{\prime}\right)=\frac{h^{\prime} \frac{1}{h^{\prime}-1}}{e \log h^{\frac{1}{h^{\prime}-1}}}, h=\frac{M}{m}, h^{\prime}=\frac{M^{\prime}}{m^{\prime}}$ and $r=\min \{\alpha, 1-\alpha\}$.

Proof. Inequality (11) is equivalent to

$$
\left\|\left[\Phi\left(A \nabla_{\alpha} B\right)+M m\left(\Phi\left(A^{-1} \nabla_{\alpha} B^{-1}\right)-S\left(h^{\prime r}\right) \Phi\left(A^{-1} \sharp_{\alpha} B^{-1}\right)\right)\right] \Phi^{-1}\left(A \sharp_{\alpha} B\right)\right\| \leq \frac{K(h)}{S\left(h^{\prime r}\right)} .
$$

By inequalities (7) and (8), we have

$$
\begin{aligned}
& \left\|\left[\Phi\left(A \nabla_{\alpha} B\right)+M m\left(\Phi\left(A^{-1} \nabla_{\alpha} B^{-1}\right)-S\left(h^{\prime r}\right) \Phi\left(A^{-1} \sharp_{\alpha} B^{-1}\right)\right)\right] M m S\left(h^{\prime r}\right) \Phi^{-1}\left(A \sharp_{\alpha} B\right)\right\| \\
& \leq \frac{1}{4}\left\|\left[\Phi\left(A \nabla_{\alpha} B\right)+M m\left(\Phi\left(A^{-1} \nabla_{\alpha} B^{-1}\right)-S\left(h^{\prime r}\right) \Phi\left(A^{-1} \sharp_{\alpha} B^{-1}\right)\right)\right]+M m S\left(h^{\prime r}\right) \Phi^{-1}\left(A \sharp_{\alpha} B\right)\right\|^{2} \\
& \leq \frac{1}{4}\left\|\left[\Phi\left(A \nabla_{\alpha} B\right)+M m\left(\Phi\left(A^{-1} \nabla_{\alpha} B^{-1}\right)-S\left(h^{\prime r}\right) \Phi\left(A^{-1} \sharp_{\alpha} B^{-1}\right)\right)\right]+M m S\left(h^{\prime r}\right) \Phi\left(\left(A \sharp_{\alpha} B\right)^{-1}\right)\right\|^{2} \\
& \leq \frac{1}{4}\left\|\Phi\left(A \nabla_{\alpha} B\right)+M m \Phi\left(A^{-1} \nabla_{\alpha} B^{-1}\right)\right\|^{2} \\
& \leq \frac{1}{4}(M+m)^{2} .
\end{aligned}
$$

That is

$$
\left\|\left[\Phi\left(A \nabla_{\alpha} B\right)+M m\left(\Phi\left(A^{-1} \nabla_{\alpha} B^{-1}\right)-S\left(h^{\prime r}\right) \Phi\left(A^{-1} \sharp_{\alpha} B^{-1}\right)\right)\right] \Phi^{-1}\left(A \sharp_{\alpha} B\right)\right\| \leq \frac{K(h)}{S\left(h^{\prime r}\right)} .
$$

Thus, inequality (11) holds. 
Inequality (12) is equivalent to

$$
\left\|\left[\Phi\left(A \nabla_{\alpha} B\right)+M m\left(\Phi\left(A^{-1} \nabla_{\alpha} B^{-1}\right)-S\left(h^{\prime r}\right) \Phi\left(A^{-1}\right) \sharp_{\alpha} \Phi\left(B^{-1}\right)\right)\right]\left[\Phi(A) \sharp_{\alpha} \Phi(B)\right]^{-1}\right\| \leq \frac{K(h)}{S\left(h^{\prime r}\right)} .
$$

By inequalities (7) and (8), we have

$$
\begin{aligned}
& \left\|\left[\Phi\left(A \nabla_{\alpha} B\right)+M m\left(\Phi\left(A^{-1} \nabla_{\alpha} B^{-1}\right)-S\left(h^{\prime r}\right) \Phi\left(A^{-1}\right) \sharp_{\alpha} \Phi\left(B^{-1}\right)\right)\right] M m S\left(h^{\prime r}\right)\left[\Phi(A) \sharp_{\alpha} \Phi(B)\right]^{-1}\right\| \\
& \leq \frac{1}{4}\left\|\Phi\left(A \nabla_{\alpha} B\right)+M m\left(\Phi\left(A^{-1} \nabla_{\alpha} B^{-1}\right)-S\left(h^{\prime r}\right) \Phi\left(A^{-1}\right) \sharp_{\alpha} \Phi\left(B^{-1}\right)\right)+M m S\left(h^{\prime r}\right)\left[\Phi(A) \sharp_{\alpha} \Phi(B)\right]^{-1}\right\|^{2} \\
& \leq \frac{1}{4}\left\|\Phi\left(A \nabla_{\alpha} B\right)+M m\left(\Phi\left(A^{-1} \nabla_{\alpha} B^{-1}\right)-S\left(h^{\prime r}\right) \Phi\left(A^{-1}\right) \sharp_{\alpha} \Phi\left(B^{-1}\right)\right)+M m S\left(h^{\prime r}\right) \Phi\left(A^{-1}\right) \sharp_{\alpha} \Phi\left(B^{-1}\right)\right\|^{2} \\
& \leq \frac{1}{4}\left\|\Phi\left(A \nabla_{\alpha} B\right)+M m \Phi\left(A^{-1} \nabla_{\alpha} B^{-1}\right)\right\|^{2} \\
& \leq \frac{1}{4}(M+m)^{2} .
\end{aligned}
$$

That is

$$
\left\|\left[\Phi\left(A \nabla_{\alpha} B\right)+M m\left(\Phi\left(A^{-1} \nabla_{\alpha} B^{-1}\right)-S\left(h^{\prime r}\right) \Phi\left(A^{-1}\right) \sharp_{\alpha} \Phi\left(B^{-1}\right)\right)\right]\left[\Phi(A) \sharp_{\alpha} \Phi(B)\right]^{-1}\right\| \leq \frac{K(h)}{S\left(h^{\prime r}\right)} .
$$

Thus, inequality (12) holds.

Remark 1. Because of inequality (10), inequalities (11) and (12) are sharper than inequalities (5) and (6), respectively.

In what follows, when $\alpha=\frac{1}{2}$, we present an example showing that inequalities (11) and (12) are sharper than inequalities (5) and (6), respectively.

Example 1. Take $A=\left[\begin{array}{ll}1 & 0 \\ 0 & 1\end{array}\right], B=\left[\begin{array}{ll}3 & 0 \\ 0 & 3\end{array}\right], \Phi(X)=X, m=\frac{1}{2}, m^{\prime}=2, M^{\prime}=\frac{5}{2}, M=4$. For the left side of inequalities (5) and (6),

$$
\left(\frac{A+B}{2}\right)^{2}=\left[\begin{array}{ll}
4 & 0 \\
0 & 4
\end{array}\right] .
$$

For the left side of inequalities (11) and (12),

$$
\left(\frac{A+B}{2}+2\left(\frac{A^{-1}+B^{-1}}{2}-S\left(\frac{\sqrt{5}}{2}\right) A^{-1} \sharp_{\frac{1}{2}} B^{-1}\right)\right)^{2} \approx\left[\begin{array}{cc}
4.7382 & 0 \\
0 & 4.7382
\end{array}\right] .
$$

In the next theorem, we show new weighted arithmetic-geometric operator mean inequalities which generalize inequalities (3) and (4).

Theorem 2. Assume $A, B>0$ and let $\Phi$ be a positive unital linear map. If $0<m \leq A \leq m^{\prime}<M^{\prime} \leq B \leq M$ or $0<m \leq B \leq m^{\prime}<M^{\prime} \leq A \leq M$ and $2<p<\infty$, then for any $\alpha \in[0,1]$,

$$
\begin{aligned}
& {\left[\Phi\left(A \nabla_{\alpha} B\right)+M m\left(\Phi\left(A^{-1} \nabla_{\alpha} B^{-1}\right)-S\left(h^{\prime r}\right) \Phi\left(A^{-1} \sharp_{\alpha} B^{-1}\right)\right)\right]^{2 p}} \\
& \leq \frac{1}{16}\left[\frac{4 K(h)}{S\left(h^{\prime r}\right)}\right]^{2 p} \Phi^{2 p}\left(A \sharp_{\alpha} B\right)
\end{aligned}
$$


and

$$
\begin{aligned}
& {\left[\Phi\left(A \nabla_{\alpha} B\right)+M m\left(\Phi\left(A^{-1} \nabla_{\alpha} B^{-1}\right)-S\left(h^{\prime r}\right) \Phi\left(A^{-1}\right) \sharp_{\alpha} \Phi\left(B^{-1}\right)\right)\right]^{2 p}} \\
& \leq \frac{1}{16}\left[\frac{4 K(h)}{S\left(h^{\prime r}\right)}\right]^{2 p}\left[\Phi(A) \sharp_{\alpha} \Phi(B)\right]^{2 p},
\end{aligned}
$$

where $S\left(h^{\prime}\right)=\frac{h^{\prime} \frac{1}{h^{\prime}-1}}{e \log h^{\prime} h^{\prime}-1}, h=\frac{M}{m}, h^{\prime}=\frac{M^{\prime}}{m^{\prime}}$ and $r=\min \{\alpha, 1-\alpha\}$.

Proof. Inequality (13) is equivalent to

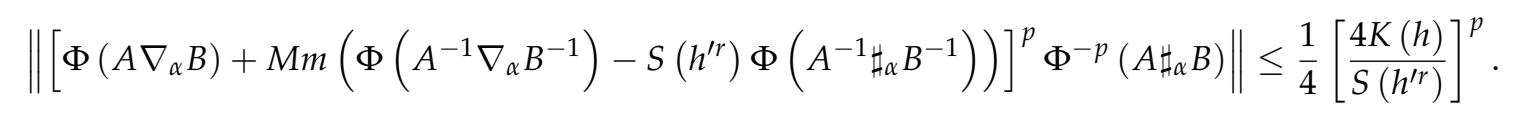

Let $L=\Phi\left(A \nabla_{\alpha} B\right)+M m \Phi\left(A^{-1} \nabla_{\alpha} B^{-1}\right)$.

By inequalities (7)-(9), we have

$$
\begin{aligned}
& \left\|\left[L-M m S\left(h^{\prime r}\right) \Phi\left(A^{-1} \sharp_{\alpha} B^{-1}\right)\right]^{p} M^{p} m^{p} S^{p}\left(h^{\prime r}\right) \Phi^{-p}\left(A \sharp_{\alpha} B\right)\right\| \\
& \leq \frac{1}{4}\left\|\left[L-M m S\left(h^{\prime r}\right) \Phi\left(A^{-1} \sharp_{\alpha} B^{-1}\right)\right]^{p}+M^{p} m^{p} S^{p}\left(h^{\prime r}\right) \Phi^{-p}\left(A \sharp_{\alpha} B\right)\right\|^{2} \\
& \leq \frac{1}{4}\left\|L-M m S\left(h^{\prime r}\right) \Phi\left(A^{-1} \sharp_{\alpha} B^{-1}\right)+M m S\left(h^{\prime r}\right) \Phi^{-1}\left(A \sharp_{\alpha} B\right)\right\|^{2 p} \\
& \leq \frac{1}{4}\left\|L-M m S\left(h^{\prime r}\right) \Phi\left(A^{-1} \sharp_{\alpha} B^{-1}\right)+M m S\left(h^{\prime r}\right) \Phi\left(\left(A \sharp_{\alpha} B\right)^{-1}\right)\right\|^{2 p} \\
& \leq \frac{1}{4}(M+m)^{2 p} .
\end{aligned}
$$

That is

$$
\left\|\left[\Phi\left(A \nabla_{\alpha} B\right)+M m\left(\Phi\left(A^{-1} \nabla_{\alpha} B^{-1}\right)-S\left(h^{\prime r}\right) \Phi\left(A^{-1} \sharp_{\alpha} B^{-1}\right)\right)\right]^{p} \Phi^{-p}\left(A \sharp_{\alpha} B\right)\right\| \leq \frac{1}{4}\left[\frac{4 K(h)}{S\left(h^{\prime r}\right)}\right]^{p} .
$$

Thus, inequality (13) holds.

Similarly, inequality (14) holds.

Theorem 3. Let all the assumptions of Theorem 1 hold. Then, for any $\alpha \in[0,1]$,

$$
\begin{aligned}
& {\left[\Phi\left(A \nabla_{\alpha} B\right)+M m\left(\Phi\left(A^{-1} \nabla_{\alpha} B^{-1}\right)-S\left(h^{\prime r}\right) \Phi\left(A^{-1} \sharp_{\alpha} B^{-1}\right)\right)\right]^{p}} \\
& \leq\left\{\begin{array}{l}
{\left[\frac{K(h)}{S\left(h^{\prime r}\right)}\right]^{p} \Phi^{p}\left(A \sharp_{\alpha} B\right), 0 \leq p \leq 2} \\
\frac{1}{4}\left[\frac{4 K(h)}{S\left(h^{\prime r}\right)}\right]^{p} \Phi^{p}\left(A \sharp_{\alpha} B\right), p>2
\end{array}\right.
\end{aligned}
$$

and

$$
\begin{aligned}
& {\left[\Phi\left(A \nabla_{\alpha} B\right)+M m\left(\Phi\left(A^{-1} \nabla_{\alpha} B^{-1}\right)-S\left(h^{\prime r}\right) \Phi\left(A^{-1}\right) \sharp_{\alpha} \Phi\left(B^{-1}\right)\right)\right]^{p}} \\
& \leq\left\{\begin{array}{l}
{\left[\frac{K(h)}{S\left(h^{\prime r}\right)}\right]^{p}\left[\Phi(A) \sharp_{\alpha} \Phi(B)\right]^{p}, 0 \leq p \leq 2} \\
\frac{1}{4}\left[\frac{4 K(h)}{S\left(h^{\prime r}\right)}\right]^{p}\left[\Phi(A) \sharp_{\alpha} \Phi(B)\right]^{p}, p>2 .
\end{array}\right.
\end{aligned}
$$


Proof. It is well known that $t^{v}(0 \leq v \leq 1)$ is an operator monotone function. Applying $t^{\frac{p}{2}}(0 \leq p \leq 2)$ and $t^{\frac{1}{2}}$ to inequalities (11) and (13), respectively, we have inequality (15).

Similarly, inequality (16) holds.

\section{Conclusions}

In this paper, we first present two weighted arithmetic-geometric operator mean inequalities, which refine and generalize inequalities (5) and (6), moreover, an example shows that inequalities (11) and (12) are sharper than inequalities (5) and (6), respectively. Finally, we generalize inequalities (11) and (12) to the power of $p(p \geq 2)$, which refine inequalities (3) and (4).

Author Contributions: The authors contributed equally to the manuscript. Both authors read and approved the final manuscript.

Funding: This research was funded by the Scientific Research Fund of Yunnan Provincial Education Department (Grant No. 2019J0350).

Acknowledgments: The authors wish to express their heartfelt thanks to the referees for their constructive comments and suggestions for revising the manuscript.

Conflicts of Interest: The authors declare no conflict of interest.

\section{References}

1. Lin, M. On an operator Kantorovich inequality for positive linear maps. J. Math. Anal. Appl. 2013, 402, 127-132. [CrossRef]

2. Lin, M. Squaring a reverse AM-GM inequality. Stud. Math. 2013, 215, 187-184. [CrossRef]

3. Zhang, P. More operator inequalities for positive linear maps. Banach J. Math. Anal. 2015, 9, 166-172. [CrossRef]

4. Xue, J. Weighted arithmetic-geometric operator mean inequalities. J. Inequal. Appl. 2018, 2018, 154. [CrossRef]

5. Yang, C.; Lu, F. Improving some operator inequalities for positive linear maps. Filomat 2018, 32, 4333-4340. [CrossRef]

6. Zou, L. An arithmetic-geometric mean inequality for singular values and its applications. Linear Algebra Appl. 2017, 528, 25-32. [CrossRef]

7. Zou, L. Unification of the arithmetic-geometric mean and Hölder inequalities for unitarily invariant norms. Linear Algebra Appl. 2019, 562, 154-162. [CrossRef]

8. Kittaneh, F.; Krnić, M. Refined Heinz operator inequalities. Linear Multilinear Algebra 2013, 61, 1148-1157. [CrossRef]

9. Gümüs, I.H.; Moradi, H.R.; Sababheh, M. More accurate operator means inequalities. J. Math. Anal. Appl. 2018, 465, 267-280. [CrossRef]

10. Bhatia, R.; Kittaneh, F. Notes on matrix arithmetic-geometric mean inequalities. Linear Algebra Appl. 2000, 308, 203-211. [CrossRef]

11. Bhatia, R. Positive Definite Matrices; Princeton University Press: Princeton, NJ, USA, 2007.

12. Ando, T.; Zhan, X. Norm inequalities related to operator monotone functions. Math. Ann. 1999, 315, 771-780. [CrossRef]

13. Zuo, H.; Shi, G.; Fujii, M. Refined Young inequality with Kantorovich constant. J. Math. Inequal. 2011, 5, 551-556. [CrossRef]

(C) 2019 by the authors. Licensee MDPI, Basel, Switzerland. This article is an open access article distributed under the terms and conditions of the Creative Commons Attribution (CC BY) license (http:// creativecommons.org/licenses/by/4.0/). 